
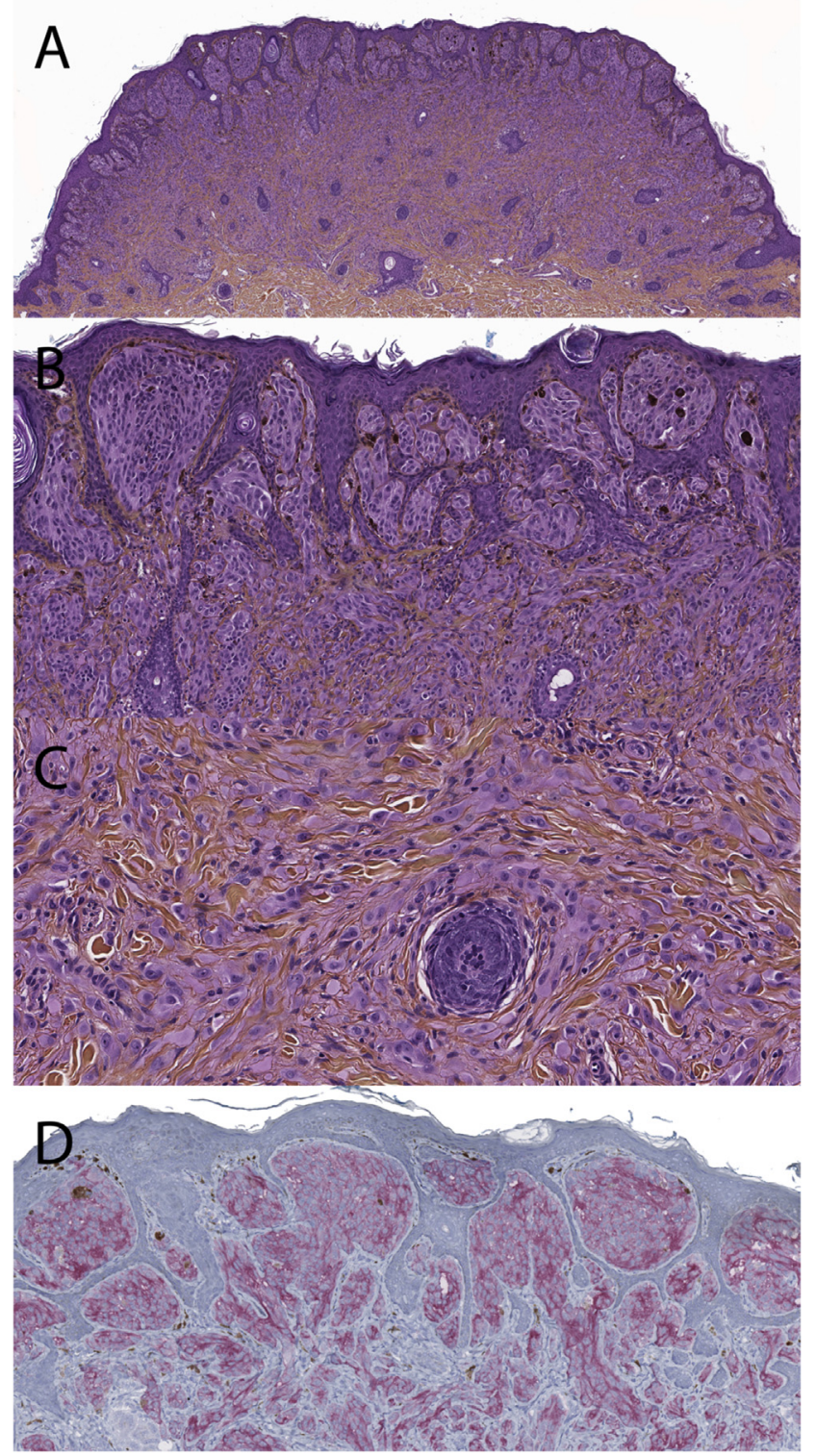

Fig. 2 (A-C) Microscopic and (D) immunohistochemical features of the Spitz naevus. (A-C) Microscopic examination revealed a dermal plexiform proliferation of spindle non-atypical melanocytes entrapped in a desmoplastic stroma (D) Immunohistochemistry demonstrated the RAS Q61R mutant in tumour cells

To conclude, our observation reports that in addition to G13R HRAS mutations, ROS1 and $L C K$ fusions, a postzygotic activating Q61R HRAS mutation in a melanocytic progenitor might constitute a preliminary step to ASN development.

Acknowledgement: The parents of the patient gave written informed consent for publication of this case and clinical photographs.

Conflicts of interest and sources of funding: The authors state that they have no significant relationships with, or financial interest in, any commercial companies pertaining to this study and there are no conflicts of interest to disclose.

Thibault Kervarrec $^{1,2, *}$, Clémence Briand ${ }^{3, *}$, Daniel Pissaloux ${ }^{1,4}$, Franck Tirode ${ }^{4}$, Claire AbasqThomas $^{3}$, Sylvie Fraitag ${ }^{5}$, Arnaud de la Fouchardière ${ }^{1,4}$
${ }^{1}$ Department of Pathology, Centre Hospitalier Universitaire de Tours, Tours, France; ${ }^{2}$ Department of Biopathology, Center Léon Bérard, Lyon, France; ${ }^{3}$ Department of Dermatology, Centre Hospitalier Universitaire de Brest, Brest, France; ${ }^{4}$ Université de Lyon, Université Claude Bernard Lyon 1, INSERM 1052, CNRS 5286, Centre Léon Bérard, Cancer Research Center of Lyon, Equipe Labellisée Ligue contre le Cancer, Lyon, France; ${ }^{5}$ Department of Pathology, Institut Imagine, APHP, Hôpital Universitaire Necker-Enfants Malades, Université Paris Descartes, Sorbonne Paris Cité, Paris, France; "These authors contributed equally

Contact: Dr Thibault Kervarrec.

E-mail: thibaultkervarrec@yahoo.fr

1. Elder DE, Massi D, Scolyer RA, Willemze R, editors. WHO Classification of Skin Tumours. 4th ed. Lyon: IARC, 2018.

2. Wiesner T, He J, Yelensky R, et al. Kinase fusions are frequent in Spitz tumours and spitzoid melanomas. Nat Commun 2014; 5: 3116.

3. Yeh I, Busam KJ, McCalmont TH, et al. Filigree-like rete ridges, lobulated nests, rosette-like structures, and exaggerated maturation characterize Spitz tumors with NTRK1 fusion. Am J Surg Pathol 2019; 43: 737-46.

4. Houlier A, Pissaloux D, Masse I, et al. Melanocytic tumors with MAP3K8 fusions: report of 33 cases with morphological-genetic correlations. Mod Pathol 2020; 33: 846-57.

5. Goto K, Pissaloux D, Durand L, Tirode F, Guillot B, de la Fouchardière A. Novel three-way complex rearrangement of TRPM1 PUM1-LCK in a case of agminated Spitz nevi arising in a giant congenital hyperpigmented macule. Pigment Cell Melanoma Res 2020 33: $767-72$

6. Yeh I, de la Fouchardiere A, Pissaloux D, et al. Clinical, histopathologic, and genomic features of Spitz tumors with ALK fusions. Am J Surg Pathol 2015: 39: 581-91.

7. Bastian BC, LeBoit PE, Pinkel D. Mutations and copy number increase of HRAS in Spitz nevi with distinctive histopathological features. Am J Pathol 2000; 157: 967-72.

8. Zayour M, Bolognia JL, Lazova R. Multiple Spitz nevi: a clinicopathologic study of 9 patients. J Am Acad Dermatol 2012; 67: 451-8 e2.

9. Sarin KY, Sun BK, Bangs CD, et al. Activating HRAS mutation in agminated Spitz nevi arising in a nevus spilus. JAMA Dermatol 2013 149: $1077-81$.

10. Pontoizeau J, Stefan A, Comoz F, et al. Agminated Spitz nevus arising in normal skin with redundant HRAS mutation. Eur J Dermatol 2017; 27: $73-4$.

11. Sarin KY, McNiff JM, Kwok S, Kim J, Khavari PA. Activating HRAS mutation in nevus spilus. J Invest Dermatol 2014; 134 1766-8.

12. Goto K, Pissaloux D, Kauer F, Huriet V, Tirode F, de la Fouchardière A. GOPC-ROS1 mosaicism in agminated Spitz nevi: report of two cases. Virchows Arch 2021; Mar 17: https://doi.org/10.1007/s00428-02002992-5.

DOI: https://doi.org/10.1016/j.pathol.2021.04.013

\section{SMARCA4-deficient anaplastic carcinoma arising in a primary retroperitoneal mucinous adenocarcinoma}

Sir,

Primary mucinous tumours arising in the retroperitoneum are uncommon and approximately half are histologically malignant, the remainder showing benign or borderline appearances according to ovarian-type criteria. ${ }^{1}$ As with primary ovarian neoplasms, retroperitoneal mucinous 
tumours are rarely associated with foci of anaplastic carcinoma. ${ }^{1,2}$ Recently, Chaudet et al. reported expression of SWI/SNF complex proteins SMARCB1 (INI1), SMARCA4 (BRG1), SMARCA2 (BRM), and ARID1A (BAF250A) in a series of 22 ovarian and three retroperitoneal mucinous tumours with foci of anaplastic carcinoma. ${ }^{3}$ Nine cases $(36 \%)$, including one of the three retroperitoneal tumours, showed loss of expression of one or more proteins that was restricted to the anaplastic tumour component. Herein, we describe only the second case of a retroperitoneal mucinous adenocarcinoma with foci of anaplastic carcinoma demonstrating SWI/SNF complex (specifically SMARCA4)-deficiency.

A 31-year-old with no significant medical history presented with abdominal distension and bloating. Abdominal ultrasound revealed a predominantly cystic retroperitoneal mass. Serological tumour markers were within the normal range. Since initially there was a low suspicion of malignancy, elective resection was planned. However, the patient represented with acute abdominal pain requiring emergency laparotomy, which revealed a solid and cystic retroperitoneal mass (size up to $240 \mathrm{~mm}$ ) that ruptured intraoperatively. The gynaecological organs were separate from the tumour and appeared normal macroscopically. Histological examination showed a moderately differentiated mucinous carcinoma with expansile-type invasion arising on a background of a benign and borderline mucinous tumour (Fig. 1A). Shortly thereafter the patient underwent completion and staging surgery including hysterectomy, bilateral salpingo-oophorectomy with en bloc resection of an adherent $80 \mathrm{~mm}$ left para-colic mass, omentectomy, appendectomy, and left para-colic gutter and para-colic fascial biopsies.
Histology of the para-colic mass and the separate left paracolic gutter biopsy from the second operation revealed an invasive mucinous carcinoma associated with prominent dissecting extracellular mucin against a background of reactive stromal changes. In addition, both specimens showed multiple discrete tumour nodules, individually up to $6 \mathrm{~mm}$ in diameter, that were composed of solid sheets of cells with predominantly epithelioid and rhabdoid morphology and more focal spindled appearances (Fig. 1B). The nuclei were relatively uniform but with high-grade cytological appearances including prominent mitotic activity, apoptosis and focal necrosis (Fig. 1C). This anaplastic tumour component demonstrated diffuse expression of broad spectrum cytokeratin (clone MNF116) and more focal cytokeratin 7 staining. ARID1A and SMARCB1 expression was intact but there was complete loss of SMARCA4 staining (Fig. 1D). In contrast, the differentiated mucinous tumour showed retained expression of all tested SWI/SNF proteins. Both tumour components demonstrated retained expression of the mismatch repair proteins MLH1, PMS2, MSH2 and MSH6. The ovaries demonstrated normal parenchymal appearances but there were microscopic foci of differentiated mucinous carcinoma within capsular fibrous adhesions. Peritoneal fluid cytology was positive for mucinous carcinoma but the remaining surgical specimens showed benign appearances.

The histogenesis of primary retroperitoneal mucinous neoplasia is uncertain with proposed origins including teratoma, urogenital remnants, intestinal duplication, heterotopic ovarian tissue and coelomic metaplasia. ${ }^{4}$ A rare phenomenon, more widely recognised in the ovary, ${ }^{5}$ is the development of anaplastic carcinoma often in the form of a mural nodule within a cyst. Comparative genomic analysis has demonstrated a clonal relationship between the differentiated and
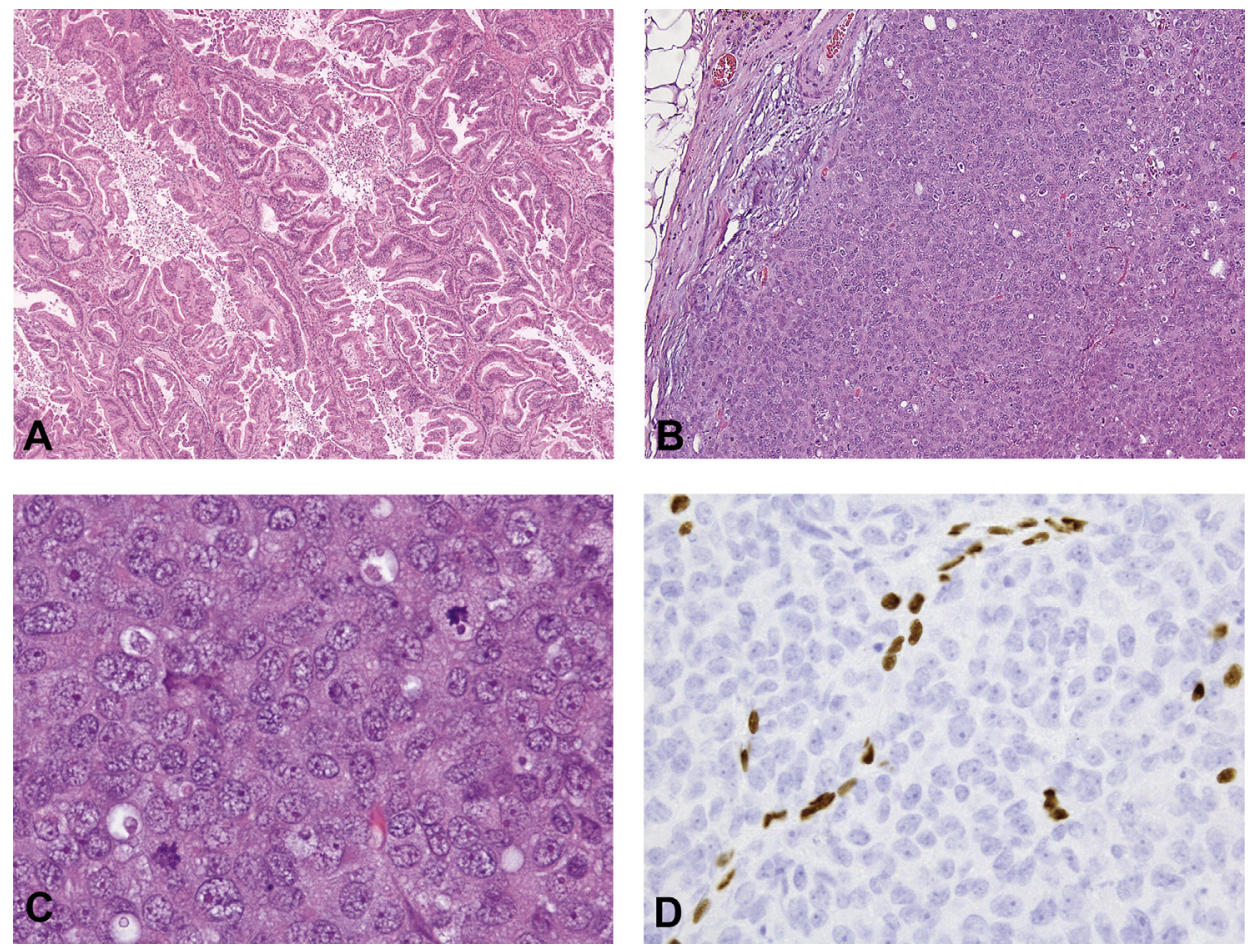

Fig. 1 (A) Mucinous carcinoma in the initial retroperitoneal mass specimen demonstrating complex glandular and papillary architecture. (B) The anaplastic carcinoma component identified at the second operation shows diffuse cellular architecture, high-grade cytology with numerous mitoses (C), and loss of SMARCA4 expression (D). Note positive internal control staining of endothelial cells. 
anaplastic tumour components in such tumours arising in the ovary. ${ }^{6}$ As in the ovary, mural nodules within retroperitoneal mucinous neoplasms can also show sarcoma-like, true sarcomatous or carcinosarcomatous histological appearances. ${ }^{4}$ In the present case, the anaplastic carcinoma component did not form a discrete nodule but rather showed multifocal distribution within areas of conventional mucinous adenocarcinoma.

The anaplastic carcinoma presented here showed loss of SMARCA4 expression similar to one of the three retroperitoneal mucinous carcinomas studied by Chaudet et ll $^{3}$ That tumour arose in a 69-year-old and showed pleomorphic morphology. There was also co-loss of SMARCA2 in the anaplastic component (not investigated in the current case) with retained SMARCB1 and ARID1A expression. Interestingly, some of the ovarian anaplastic carcinomas in that series demonstrated rhabdoid appearances, a morphological feature associated with SWI/SNF deficiency in a variety of tumours.

In summary, we report a second example of SMARCA4deficient anaplastic carcinoma arising within a retroperitoneal mucinous adenocarcinoma. We believe that this case lends support to the proposal by Chaudet and colleagues that SWI/SNF complex inactivation may be a key factor in the development of morphological anaplasia in these tumours, and more generally of 'dedifferentiation' within a range of gynaecological and gynaecological-related neoplasms.

Conflicts of interest and sources of funding: The authors state that there are no conflicts of interest to disclose.

\section{Daniel Ching ${ }^{1}$, Sukeerat Ruba ${ }^{1}$, Anita Soma ${ }^{1}$, Yee Chit Leung $^{2,3}$, Colin J. R. Stewart ${ }^{1,4}$}

${ }^{1}$ Department of Histopathology, King Edward Memorial Hospital, Perth, WA, Australia; ${ }^{2}$ Department of GynaeOncology, King Edward Memorial Hospital, Perth, WA, Australia; ${ }^{3}$ The University of Western Australia Health and Medical Sciences Division of Obstetrics and Gynaecology, Perth, WA, Australia; ${ }^{4}$ School of Women's and Infants' Health, University of Western Australia, Perth, WA, Australia

Contact Dr D. Ching.

E-mail: Daniel.ching@health.wa.gov.au

1. Roma AA, Malpica A. Primary retroperitoneal mucinous tumors: clinicopathologic study of 18 cases. Am J Surg Pathol 2009; 33: 526-33.

2. Mikami M, Tei C, Takehara K, et al. Retroperitoneal primary mucinous adenocarcinoma with a mural nodule of anaplastic tumor: a case report and literature review. Int J Gynecol Pathol 2003; 22: 205-8.

3. Chaudet K, Kem M, Lerwill M, et al. SWI/SNF protein and claudin-4 expression in anaplastic carcinomas arising in mucinous tumours of the ovary and retroperitoneum. Histopathology 2020; 77: 231-9.

4. Liu L, Zhao Q, Zhang J, et al. Primary retroperitoneal mucinous cystadenocarcinoma: a case report and literature review. Int J Clin Exp Med 2016; 9: 5318-25.

5. Provenza C, Young RH, Prat J. Anaplastic carcinoma in mucinous ovarian tumours: a clinicopathologic study of 34 cases emphasizing the crucial impact of stage on prognosis, the histologic spectrum, and overlap with sarcomalike mural nodules. Am J Surg Pathol 2008; 32: 383-9.

6. Ardakani NM, Giardina T, Amanual B, et al. Molecular profiling reveals a clonal relationship between ovarian mucinous tumors and correspond ing mural carcinomatous nodules. Am J Surg Pathol 2017; 41: 1261-6.

7. McCluggage WG, Stewart CJR. SWI/SNF-deficient malignancies of the female genital tract. Semin Diagn Pathol 2021; 38: 199-211.

DOI: https://doi.org/10.1016/j.pathol.2021.06.118

\section{Ductal-type mesonephric duct/ remnant hyperplasia: distinguished from lobular or diffuse mesonephric hyperplasia by the presence of a myoepithelial cell layer and micropapillary tufting}

\section{Sir,}

Mesonephric (Wolffian) ducts regress during normal female development. Their remnants may be found incidentally within the cervix, adnexa, vagina and uterus. ${ }^{1,2}$ These remnants may in turn appear hyperplastic, and three forms of mesonephric duct hyperplasia have been recognised: lobular, diffuse and ductal-type. ${ }^{2,3}$ The lobular and diffuse types have been well characterised in large case series. ${ }^{1,2}$ However, ductal-type mesonephric remnant hyperplasia is still poorly characterised, with only two cases described in Ferry and Scully's 1990 series of 49 cases of mesonephric duct hyperplasia. $^{2}$ This ductal type is characterised by a bilayered ductular proliferation with inner luminal cylindrical cells and outer flattened myoepithelial cells. We herein report the histopathological and immunophenotypic findings of two cases of ductal-type mesonephric remnant hyperplasia of the female genital tract.

Case 1 (Fig. 1) was a consultation biopsy specimen of a vaginal polypoid lesion from a 92-year-old otherwise asymptomatic woman, measuring $8 \times 7 \times 4 \mathrm{~mm}$. Microscopic examination revealed a normal vaginal non-keratinising squamous mucosa, with a stromal glandular proliferation arrayed in lobules. Large, dilated ducts filled with proteinaceous secretions were mainly present in the centre of the lesion, with smaller non-dilated ducts arrayed in the periphery. The lesional stroma was hypercellular when compared to the adjacent stroma. The ducts consisted of two distinct cell layers. Small, flattened outer myoepithelial cells encircled an inner layer of larger cuboidal to cylindrical, non-ciliated, bland luminal cells. The cytoplasm of these inner ductular epithelial cells was moderately abundant, pale and eosinophilic. Mitoses were absent and nucleoli were small. Some ducts showed micropapillary inward tufting towards the duct lumen. The immunophenotype of this ductal lesion demonstrated both inner epithelial and outer myoepithelial cells, with some overlapping markers (Table 1). EMA was found to be expressed only in the luminal cells, whilst p40 and p63 were exclusive to the myoepithelial cell layer.

Case 2 (Fig. 2) was a 24-year-old non-smoking G1P1 woman, with a history of recurring high-grade squamous intraepithelial lesions (HSIL) for the previous 2 years. Her loop electrosurgical excision procedure (LEEP) specimen demonstrated a low-grade squamous intraepithelial lesion, endocervical gland hyperplasia, and an incidental focus of dilated relatively deep stromal glands measuring $6 \mathrm{~mm}$ in greatest diameter. These dilated, microcystic glandular foci were composed of at least two cell layers. The outer myoepithelial layer demonstrated small, cuboidal, hyperchromatic cells, some with flattened nuclei. The inner luminal layer was formed by cylindrical, pseudostratified, at times ciliated, cells. Apical mucin was absent. Some ducts showed inward micropapillary tufting, and proteinaceous secretion materials. The immunophenotype of this ductal 